\title{
Synovitis in Knee Osteoarthritis Assessed by Contrast-enhanced Magnetic Resonance Imaging (MRI) is Associated with Radiographic Tibiofemoral Osteoarthritis and MRI-detected Widespread Cartilage Damage: The MOST Study
}

\author{
Ali Guermazi, Daichi Hayashi, Frank W. Roemer, Yanyan Zhu, Jingbo Niu, Michel D. Crema, \\ M. Kassim Javaid, Monica D. Marra, John A. Lynch, George Y. El-Khoury, Yuqing Zhang, \\ Michael C. Nevitt, and David T. Felson
}

ABSTRACT. Objective. To examine the cross-sectional association of whole-knee synovitis assessed by contrast-enhanced magnetic resonance imaging (CEMRI) with radiographic tibiofemoral osteoarthritis (OA), non-CEMRI-assessed cartilage damage, and meniscal status.

Methods. Multicenter Osteoarthritis Study (MOST) is a cohort study of people with or at high risk of knee OA. Subjects are a subset of MOST who volunteered for both CEMRI and non-CEMRI. Using CEMRI, synovitis was assessed at 11 sites and graded $0-2$ at each site. Presence of "whole-knee synovitis" was defined as the synovitis score of $\geq 1$ at any site from each knee. Cartilage and meniscal damage was evaluated using non-CEMRI based on the Whole Organ MRI Score. Logistic regression was used to assess associations of synovitis with radiographic OA (Kellgren-Lawrence grade $\geq 2$ ), widespread cartilage damage, and meniscal damage, adjusting for age, sex, and body mass index (BMI). Additional analyses were performed excluding subjects who had chondrocalcinosis on radiography and those taking antiinflammatory medications.

Results. Four hundred four subjects were included (mean age $58.8 \pm 7.0 \mathrm{yrs}$, BMI $29.6 \pm 4.9 \mathrm{~kg} / \mathrm{m}^{2}$, $45.5 \%$ women). On CEMRI, the maximum synovitis score across 11 sites in each knee was 0 in 106 knees $(26.2 \%), 1$ in $135(33.4 \%)$, and 2 in $163(40.4 \%)$. Synovitis was associated with radiographic OA [adjusted OR (aOR) 3.25, 95\% CI 1.98-5.35] and widespread cartilage damage (aOR 1.91, 95\% CI 1.24-2.92). Severe meniscal damage showed a borderline significant association with synovitis (aOR $1.74,95 \%$ CI 0.99-3.04). Additional analyses as described did not notably change the results. Conclusion. CEMRI-detected synovitis is strongly associated with tibiofemoral radiographic OA and MRI-detected widespread cartilage damage. (First Release Jan 15 2014; J Rheumatol 2014;41:501-8; doi:10.3899/jrheum.130541)

Key Indexing Terms:

SYNOVITIS OSTEOARTHRITIS CARTILAGE MENISCUS MAGNETIC RESONANCE IMAGING

From the Quantitative Imaging Center (QIC), Department of Radiology, Boston University School of Medicine, Boston, MA; Department of Radiology, Bridgeport Hospital, Yale University School of Medicine, Bridgeport, CT, USA; Department of Radiology, University of Erlangen, Erlangen, Germany; Clinical Epidemiology Research and Training Unit, Boston University School of Medicine, Boston, MA, USA; Department of Radiology, Hospital do Coração (HCor) and Teleimagem, São Paulo, Brazil; Oxford UK National Institute for Health Research (NIHR) Musculoskeletal Biomedical Research Unit, Nuffield Department of Orthopaedics, Rheumatology and Musculoskeletal Sciences, University of Oxford, Oxford, UK; Department of Epidemiology and Biostatistics, University of California San Francisco, San Francisco, CA; Department of Radiology at the University of Iowa, Iowa City, IA, USA.

Supported by National Institutes of Health (NIH) grants from the National Institute on Aging (U01-AG-18947, U01-AG-18832, U01-AG-19069, and U01-AG-18820). The MOST ancillary study is supported by NIH grants R01-AR053161 and K23AR053855. Dr. Guermazi is the president of Boston Imaging Core Lab LLC (BICL), Boston, Massachusetts, USA, a company providing radiological image assessment services. He is a consultant to MerckSerono, Sanofi-Aventis, and TissueGene. Drs. Roemer,
Crema, and Marra are shareholders of BICL. Dr. Roemer is a consultant to MerckSerono and the NIH.

A. Guermazi, MD, PhD, Professor of Radiology, QIC, Department of Radiology, Boston University School of Medicine; D. Hayashi, MD, PhD, Research Assistant Professor of Radiology, QIC, Department of Radiology, Boston University School of Medicine, and Department of Radiology, Bridgeport Hospital, Yale University School of Medicine; F.W. Roemer, MD, Associate Professor of Radiology, Department of Radiology, Boston University School of Medicine, and Department of Radiology, University of Erlangen; Y. Zhu, PhD, Research Assistant Professor; J. Niu, DSc, Research Assistant Professor, Clinical Epidemiology Research and Training Unit, Boston University School of Medicine; M.D. Crema, MD, Adjunct Assistant Professor of Radiology, QIC, Department of Radiology, Boston University School of Medicine, and Department of Radiology, HCor and Teleimagem; M.K. Javaid, MBBS, PhD, Norman Collisson Senior Research Fellow and Lecturer in Metabolic Bone Disease, Oxford NIHR Musculoskeletal Biomedical Research Unit, Nuffield Department of Orthopaedics, Rheumatology and Musculoskeletal Sciences, University of Oxford; M.D. Marra, MD, Research Instructor, QIC, Department of Radiology, Boston University 
School of Medicine; J.A. Lynch, PhD, Director, MRI Quality Assurance, Department of Epidemiology and Biostatistics, University of California San Francisco; G.Y. El-Khoury, MD, Professor, Department of Radiology at the University of Iowa; Y. Zhang, ScD, Professor of Medicine and Public Health, Clinical Epidemiology Research and Training Unit, Boston University School of Medicine; M.C. Nevitt, PhD, Adjunct Professor, Division of Clinical Trials and Multicenter Studies, Department of Epidemiology and Biostatistics, University of California San Francisco; D.T. Felson, MD, MPH, Professor of Medicine and Epidemiology, Clinical Epidemiology Research and Training Unit, Boston University School of Medicine.

Address correspondence to Dr. A. Guermazi, Department of Radiology, Boston University School of Medicine, 820 Harrison Avenue, FGH Building,3rd Floor, Boston, MA 02118,USA.E-mail: guermazi@bu.edu Accepted for publication November 29, 2013.

Inflammation of the synovial membrane is characterized on magnetic resonance imaging (MRI) by thickening and enhancement after administration of intravenous contrast agents. In osteoarthritis (OA), synovitis is thought to be a secondary phenomenon related to cartilage and bone alterations and is triggered probably by release of detritus from these joint structures ${ }^{1}$. However, it has been reported that some patients with early knee OA express inflammatory markers, such as interleukin $15^{2}$, suggesting OA does involve an active inflammatory process. Moreover, there is evidence from arthroscopic and MRI-based studies showing that OA also may play a role in cartilage $\operatorname{loss}^{3,4}$. Animal studies have shown that synovial cell response plays an important role in the repair process of meniscal tear ${ }^{5}$.

Presence and severity of synovitis in knee OA can be evaluated using contrast-enhanced T1-weighted MRI $(\text { CEMRI })^{6}$. On CEMRI, synovitis can be directly visualized as the strongly enhancing lining of the knee joint cavity on postcontrast images. A comprehensive semiquantitative scoring system for synovitis in knee OA using CEMRI has been published ${ }^{7}$. Association between CEMRI-assessed synovitis and radiographic OA severity has been reported ${ }^{8,9}$. However, to our knowledge, no studies have been published reporting the association of synovitis, evaluated on CEMRI with radiographic tibiofemoral $\mathrm{OA}$, cartilage damage, and meniscal damage, all in 1 study.

Thus, our objective was to examine the association of CEMRI-assessed whole-knee synovitis with radiographic tibiofemoral $\mathrm{OA}$ as well as with cartilage damage and meniscus damage in persons with or at high risk of knee OA.

\section{MATERIALS AND METHODS}

Study design and subjects. Subjects were participants in the Multicenter Osteoarthritis Study (MOST), a prospective study of 3026 persons aged 50-79 years with a goal of identifying risk factors for incident and progressive knee OA in a sample either with OA or at high risk of developing the disease. Those considered at high risk included persons who were overweight or obese, those with knee pain, aching, or stiffness on most of the previous 30 days, a history of knee injury that made it difficult to walk for at least 1 week, or previous knee surgery. Subjects were recruited from 2 US communities, Birmingham, Alabama, and Iowa City, Iowa. Details of subject inclusion, exclusion, and recruitment have been described ${ }^{3,10}$
Subjects were excluded from MOST if they screened positive for rheumatoid arthritis, ankylosing spondylitis, psoriatic arthritis, reactive arthritis (previously known as Reiter syndrome), had renal insufficiency that required hemo- or peritoneal dialysis, a history of cancer (except for non-melanoma skin cancer), had or planned to have bilateral knee replacement surgery, were unable to walk without assistance, or were planning to move out of the area in the next 3 years. The study protocol was approved by the institutional review boards at the University of Iowa; University of Alabama, Birmingham; University of California, San Francisco; and Boston University Medical Campus; and written informed consent was obtained from all participants.

For our present study, we examined a subset of MOST subjects who volunteered to undergo a $1.5 \mathrm{~T}$ CEMRI of 1 knee at the 30-month followup clinic visit. CEMRI were obtained on 1 knee only. To choose the knee to image, radiographs were read and the knee with the lower Kellgren-Lawrence (KL) grade was selected to avoid knees with severe OA and likely co-occurrence in these knees of structural features associated with pain. While assessment of pain was not part of our study, it was part of the ancillary study of MOST using CEMRI, from which our data were derived, and therefore the same selection criteria were applied. If the grade was the same for both knees, the dominant leg (which was defined as the leg with which the subject preferred to kick a ball) was chosen. CEMRI was performed on the same day or within 30 days of non-CEMRI obtained in all MRI eligible subjects in the parent study. For subjects with renal disease, diabetes, or those over the age of 65 , serum creatinine was determined and the glomerular filtration rate (GFR) calculated before intravenous gadolinium administration. Those subjects with renal insufficiency (GFR $<30 \mathrm{ml} / \mathrm{min}$ ) were excluded from the study. No adverse events following contrast administration were reported in any subjects. Figure 1 shows the flow chart of the subject selection process.

Radiographs. All subjects underwent weight-bearing posteroanterior fixed flexion knee radiographs using the protocol by Peterfy, et al, and an acrylic positioning frame (SynaFlexer) ${ }^{11}$ at 30 -month followup. A musculoskeletal radiologist (a non-author) and a rheumatologist (DTF) blinded to clinical data graded radiographs according to the KL grade $^{12}$, followed by an adjudication process (by 2 non-authors and DTF). The weighted kappa coefficient of interobserver reliability for the KL readings of the radiograph was 0.79 .

MRI acquisition. In the MOST parent study, MR imaging without contrast enhancement was performed using a 1.0T extremity-based OrthOne scanner (GE Healthcare). Images were acquired using a circumferential extremity coil with fat-suppressed, fast spin echo, proton density-weighted sequence in 2 planes, sagittal [TR $4800 \mathrm{~ms}$, TE $35 \mathrm{~ms}, 3.0 \mathrm{~mm}$ slice thickness, $0 \mathrm{~mm}$ interslice gap, field of view (FOV) $14 \times 14 \mathrm{~cm}$, matrix 288 $\times$ 192, NEX2]; and axial (TR $4700 \mathrm{~ms}$, TE $13.2 \mathrm{~ms}, 3.0 \mathrm{~mm}$ slice thickness, $0 \mathrm{~mm}$ interslice gap, FOV $14 \mathrm{~cm}$, matrix $288 \times 192$, NEX2) and a short-tau inversion recovery sequence in the coronal plane (TR $7820 \mathrm{~ms}$, TE $14 \mathrm{~ms}$, TI $100 \mathrm{~ms}, 3.0 \mathrm{~mm}$ slice thickness, $0 \mathrm{~mm}$ interslice gap, FOV $14 \mathrm{~cm}$, matrix $256 \times 256$, NEX2)

For the purpose of our study, CEMRI were obtained with $1.5 \mathrm{~T}$ system (Siemens Symphony) with a circumferential extremity coil. Axial and sagittal fat-suppressed T1-weighted CE sequences were acquired (TR 600 $\mathrm{ms}$, TE $13 \mathrm{~ms}, 3.0 \mathrm{~mm}$ slice thickness, $0.3 \mathrm{~mm}$ interslice gap, FOV $16 \times 16$ $\mathrm{cm}$, matrix $512 \times 512$, ETL 1). Intravenous gadolinium [Magnevist (gadopentetate dimeglumine); Bayer Schering Pharma AG] or Omniscan (gadodiamide; GE Healthcare) was administered at a dose of $0.2 \mathrm{ml}(0.1$ $\mathrm{mmol} / \mathrm{kg}$ body weight. Two minutes after completing the injection of the gadolinium, sagittal sequences were obtained, immediately followed by the axial sequences. The timing of scanning ( 2 min postinjection) was chosen so that we could visualize the maximal synovial enhancement, and also to be able to complete acquisition of images before blurring of synovitis/effusion borderline occurred, caused by effusion enhancement from the periphery ${ }^{13}$. The CEMRI was performed on the same day or within 30 days of non-CEMRI scans obtained in all MRI-eligible subjects. 


\title{
Baseline: 6053 knees \\ (3026 subjects)
}

Knees without CEMRI: 5648

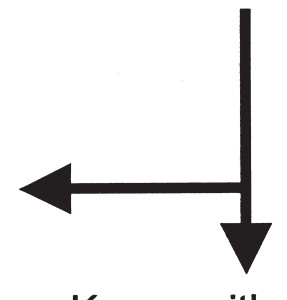

Knees with CEMRI:

405

\section{Knees without measurement of: Radiographic OA: 1 Cartilage damage: 63 Meniscal damage: 62}

\author{
Knees used in analysis for: \\ Radiographic OA (Table 1): 404 \\ Cartilage damage (Table 2): 342 \\ Meniscal damage (Table 3): 343
}

Figure 1. Flow chart showing subject inclusion and exclusion for the current study. CEMRI: contrast-enhanced magnetic resonance imaging; OA: osteoarthritis.

MRI interpretation. MRI readings were performed independently by 2 musculoskeletal radiologists (AG, FWR), with 11 and 9 years of experience, respectively, in semiquantitative MR assessment of knee OA, after an initial session of training and calibration with 20 test cases that were randomly selected. Axial and sagittal CEMRI sequences were used only for the purpose of synovitis assessment. Non-CEMRI was used for scoring of cartilage damage and meniscal damage. MR images were assessed using eFilm software (Version 2.0.0, Merge Healthcare).

We used a published scoring method for synovitis by Guermazi and colleagues $^{7}$ based on CEMRI. Using this scoring method, synovitis was defined as enhancing thickened synovium $(\geq 2 \mathrm{~mm}$ ) and was evaluated at 11 sites of the joint, i.e., the medial and lateral parapatellar recess, suprapatellar, infrapatellar, intercondylar, medial and lateral perimeniscal, and adjacent to the anterior and posterior cruciate ligaments in all subjects. If knees presented with Baker's cysts or loose bodies, those 2 sites were scored in addition. Synovial thickness was scored semiquantitatively based on the maximal thickness in any slice at each site as follows: grade 0 if $<2$ $\mathrm{mm}$, grade 1 if $2-4 \mathrm{~mm}$, and grade 2 if $>4 \mathrm{~mm}^{7}$.

Cartilage damage was semiquantitatively assessed using the Whole Organ Magnetic Resonance Imaging Score (WORMS) ${ }^{14}$. In each of the 14 articular-surface subregions defined by WORMS, cartilage was scored using an 8-point scale: $0=$ normal thickness and signal; $1=$ normal thickness but increased signal on fat-suppressed proton density-weighted images; 2 = partial-thickness focal defect $<1 \mathrm{~cm}$ in greatest width; $2.5=$ full-thickness focal defect $<1 \mathrm{~cm}$ in greatest width; $3=$ multiple areas of partial-thickness (grade 2) defects intermixed with areas of normal thickness; $4=$ diffuse $(\geq 75 \%$ of the region) partial-thickness loss; $5=$ multiple areas of full-thickness loss (grade 2.5) or a grade 2.5 lesion wider than $1 \mathrm{~cm}$ but $<75 \%$ of the region; $6=$ diffuse $(\geq 75 \%$ of the region) full-thickness loss. The number of subregions with a score of 2 or above was noted for each knee, and knees with 4 or more subregions with cartilage score of 2 or above were considered to have widespread cartilage damage. However, there is no definitive definition of "widespread cartilage damage" and the change in the threshold may produce different results. Thus, we also used an alternative definition for "widespread" cartilage damage as having cartilage score of 2 or above in 6 or more subregions.

Meniscal damage was also assessed using the WORMS system. The anterior, middle, and posterior thirds of each meniscus were scored for tears and degeneration using the following 5-point scale: $0=$ normal; $1=$ minimal radial or parrot beak tear; $2=$ non-displaced tear or surgical repair; $3=$ displaced tear or partial maceration or partial resection; $4=$ complete maceration or total resection. The maximum score of all subregions in each meniscus was recorded and used for analyses.

Statistical analysis. For the CEMRI-based assessment of synovitis, we used the maximum synovitis score (1 or above in any location) from each knee as the definition of the presence of whole-knee synovitis. Interobserver and intraobserver reliability for each individual site was calculated using weighted kappa statistics on a set of 50 randomly selected MR examinations, which were re-read with an interval $>1$ month between readings. The intraobserver reliability (weighted kappa) for the individual sites were $0.67-1.00$ for reader 1 and $0.63-1.00$ for reader 2 . Interobserver reliability for the individual sites ranged $0.67-0.92^{7}$.

Logistic regression with the proportional odds model was used to assess the possible association of the presence of definite synovitis (defined by the

Personal non-commercial use only. The Journal of Rheumatology Copyright (c) 2014. All rights reserved. 
summed synovitis score of 5 or above, as described) and the maximum synovitis score with the presence of radiographic OA (KL grade 2 or above), widespread cartilage damage (2 definitions, as defined), meniscal status (maximum WORMS score of 1 or above in any subregion), adjusting for age, sex, and body mass index (BMI).

We excluded the following subjects from these analyses: (1) those who had chondrocalcinosis on radiography, which may suggest the presence of calcium pyrophosphate deposition disease, an important concomitant condition in OA that may lead to synovitis as well as secondary OA, and (2) those who were taking antiinflammatory medication, which might have affected the severity of synovitis. All analyses were performed using SAS 9.1 (SAS Institute)

\section{RESULTS}

A total of 404 subjects were included (Table 1). The mean age was $58.8 \pm 7.0$ years, mean BMI $29.6 \pm 4.9 \mathrm{~kg} / \mathrm{m}^{2}$, and $45.5 \%$ were women. On CEMRI, the maximum synovitis score for each knee was 0 in 106 knees (26.2\%), 1 in 135 knees (33.4\%), and 2 in 163 knees $(40.4 \%)$. There was no radiographic knee OA (KL grade 0 or 1) in 318 knees (78.7\%), while $86(11.3 \%)$ knees did have it (KL grade 2, 3, or 4).

Radiographic OA was strongly associated with the presence of CEMRI-based whole-knee synovitis [adjusted OR (aOR) 3.25, 95\% CI 1.98-5.35; Figure 2]. In subjects with radiographic knee $\mathrm{OA}$, the higher the $\mathrm{KL}$ grade, the higher the likelihood of whole-knee synovitis (Table 2). Results did not notably change when we excluded subjects with chondrocalcinosis on radiography or those taking antiinflammatory medications (Table 2).

Table 1. Demographic characteristics, radiographic osteoarthritis (OA) status, and CEMRI-based synovitis grades of the study subjects.

\begin{tabular}{|c|c|}
\hline Total No. Subjects & $\mathrm{N}=404$ \\
\hline Age, yrs, mean \pm SD & $58.8 \pm 7.0$ \\
\hline $\mathrm{BMI}, \mathrm{kg} / \mathrm{m}^{2}$, mean $\pm \mathrm{SD}$ & $29.6 \pm 4.9$ \\
\hline Sex, women, n (\%) & $184(45.5)$ \\
\hline \multicolumn{2}{|l|}{ Kellgren-Lawrence grade } \\
\hline 0 or 1 & $318(78.7 \%)$ \\
\hline 2 & 41 \\
\hline 3 & 37 \\
\hline 4 & 8 \\
\hline$\geq 2$ & $86(11.3 \%)$ \\
\hline \multicolumn{2}{|l|}{ Max synovitis score on CEMRI, n (\%) } \\
\hline 0 & $106(26.2)$ \\
\hline 1 & $135(33.4)$ \\
\hline 2 & $163(40.4)$ \\
\hline Presence of cartilage damage (WORMS grade $\geq 2$ ) & $\mathrm{n}=342$ \\
\hline$<4$ subregions & 117 \\
\hline$\geq 4$ subregions & 225 \\
\hline$<6$ subregions & 208 \\
\hline$\geq 6$ subregions & 134 \\
\hline Max meniscal grade in any subregion & $\mathrm{n}=343$ \\
\hline 0 & 208 \\
\hline 1 & 20 \\
\hline 2 & 54 \\
\hline$\geq 3$ & 61 \\
\hline
\end{tabular}

CEMRI: contrast-enhanced magnetic resonance imaging; BMI: body mass index; WORMS: Whole Organ Magnetic Resonance Imaging Score.
There was also a strong association between maximum synovitis scores and widespread cartilage damage (Table 3; Figure 3). This association remained statistically significant when the alternative definition of widespread cartilage damage was used. Moreover, similar associations were observed after excluding subjects with chondrocalcinosis on radiography or those taking antiinflammatory medications (Table 3).

Only severe meniscal damage (max meniscal score $\geq 3$ ) showed a borderline significant association with maximum synovitis score (aOR 1.74, 95\% CI 0.99-3.04; Table 4). Statistically significant associations were not found regardless of the presence of chondrocalcinosis on radiography and the history of taking antiinflammatory medications.

\section{DISCUSSION}

In our study, we used a newly developed comprehensive semiquantitative scoring system based on CEMRI to assess whole-knee synovitis in knee OA. We found that whole-knee synovitis is cross-sectionally associated with radiographic knee $\mathrm{OA}$, and that the risk of synovitis increased as the severity of knee OA increased. Widespread cartilage damage of the tibiofemoral joint was also associated with whole-knee synovitis. As for meniscal damage, only severe lesions showed borderline significant association with whole-knee synovitis. We could only assess the cross-sectional association between synovitis and cartilage/meniscal damage. To investigate whether the CEMRI-detected synovitis predicts structural alterations of cartilage and meniscus, a longitudinal study is needed.

Synovitis has been shown to occur as a secondary phenomenon in the OA disease process. Release of detritus from joint structures including cartilage is thought to be its trigger $^{15}$. Macrophages in the synovial lining proliferate to phagocytose this detritus, producing a tissue whose lining becomes inflamed and thicker. Synovial activation may be caused by ligamentous injury, meniscal tears, loose bodies, or hyaline cartilage deterioration in moderate to advanced OA. Studies have reported the presence of inflammatory mediators in $\mathrm{OA}^{2,16}$ as well as a relationship between synovitis and pro-fasting plasma phospholipids that have proinflammatory effects ${ }^{17}$.

The strong association of whole-knee synovitis with radiographic OA severity was reported previously, using the summed synovitis score to represent whole-knee synovitis $^{7,9}$. In our study, we showed that a strong association can also be found when the maximum synovitis score is used as a marker of whole-knee synovitis. We also observed an increasing trend, i.e., the higher the KL grade, the higher the likelihood of whole-knee synovitis. Our findings may suggest the presence of active inflammatory processes in endstage knee OA (KL grade 4).

Based on non-CEMRI findings, a study by Meredith, et al reported that the association between semiquantitatively

Personal non-commercial use only. The Journal of Rheumatology Copyright $@$ 2014. All rights reserved. 

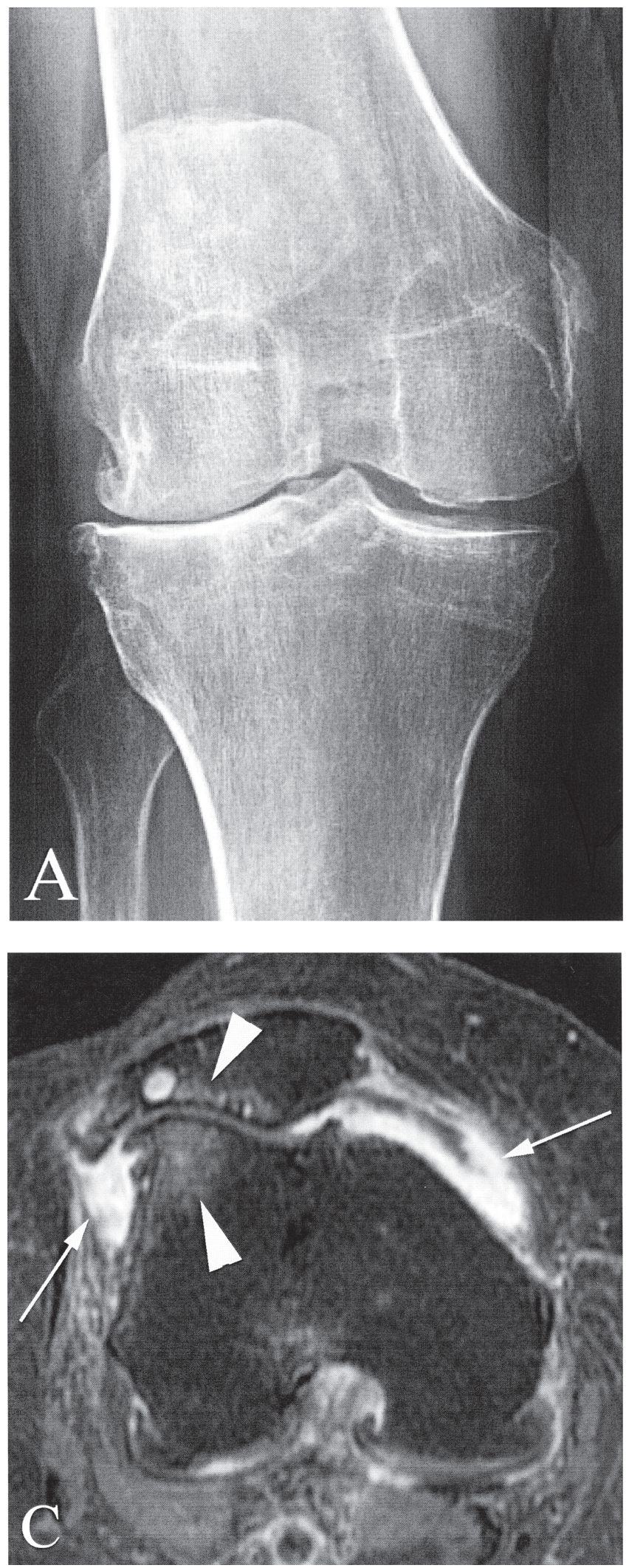

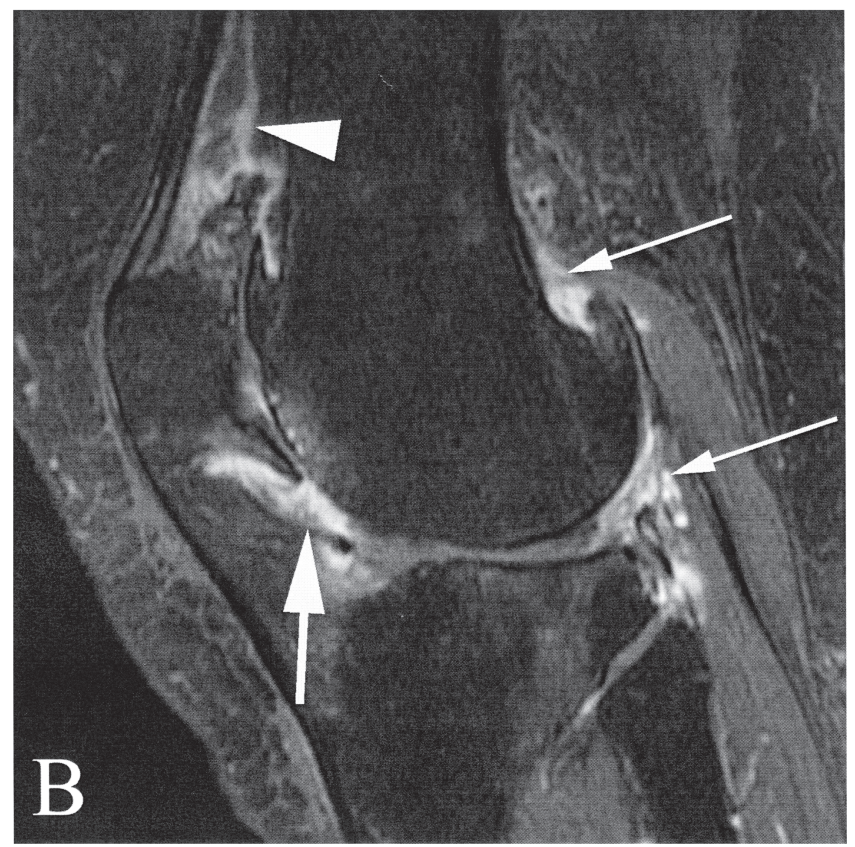

Figure 2. Severe cartilage loss in the lateral tibiofemoral and patellofemoral compartments. A. Posteroanterior radiograph of the right knee shows diffuse medial and lateral tibiofemoral osteophytosis and lateral joint space narrowing, which classify the knee as Kellgren-Lawrence grade 3. B. Sagittal contrast-enhanced T1-weighted magnetic resonance imaging (MRI) shows marked synovitis at the dorsal surface of Hoffa's fat pad (large arrow). In addition there is severe synovitis at the suprapatellar recess (arrowhead) and posteriorly adjacent to a femoral osteophyte and perimensical (thin arrows). C. Axial contrast-enhanced T1-weighted MRI shows diffuse severe synovial thickening at the medial and lateral patellar recesses (arrows). There is also diffuse full thickness cartilage loss in the lateral patellofemoral joint with adjacent bone marrow lesions (arrowheads). 
Table 2. Cross-sectional association of radiographic osteoarthritis and maximum synovitis scores from the whole knee.

\begin{tabular}{|c|c|c|c|}
\hline \multirow[b]{2}{*}{ KL Grade } & \multicolumn{3}{|c|}{ Adjusted* OR (95\% CI) for CEMRI Max Synovitis Score ${ }^{\dagger}$} \\
\hline & $\begin{array}{l}\text { All Subjects, } \\
\quad \mathrm{n}=404\end{array}$ & $\begin{array}{l}\text { Excluding Subjects Taking } \\
\text { Antiinflammatory Drugs, } \\
\qquad \mathrm{n}=347\end{array}$ & $\begin{array}{c}\text { Excluding Subjects with } \\
\text { Chondrocalcinosis on } \\
\text { Radiography, } n=374\end{array}$ \\
\hline 0 or 1 & 1.00 (reference) & 1.00 (reference) & 1.00 (reference) \\
\hline 2 & $2.34(1.22-4.50)$ & $2.42(1.21,4.86)$ & $2.27(1.14,4.55)$ \\
\hline 3 & $3.74(1.84-7.60)$ & $3.25(1.47,7.2)$ & $3.36(1.6,7.07)$ \\
\hline 4 & $15.59(1.82-133.33)$ & $14.78(1.72,126.91)$ & $13.39(1.53,117.24)$ \\
\hline$\geq 2$ & $3.25(1.98-5.35)$ & $3.13(1.82,5.39)$ & $3.06(1.81,5.16)$ \\
\hline
\end{tabular}

* Adjustment made for age, sex, and body mass index. ${ }^{\dagger}$ Synovitis score $\geq 1$ in at least 1 of 11 sites. KL: Kellgren-Lawrence; CEMRI: contrast-enhanced magnetic resonance imaging.

Table 3. Cross-sectional association of widespread cartilage damage* and maximum synovitis scores from the whole knee.

\begin{tabular}{lccc}
$\begin{array}{l}\text { Presence of } \\
\text { Cartilage Damage }\end{array}$ & $\begin{array}{c}\text { Adjusted*** OR }(95 \% \text { CI) for CEMRI Max Synovitis Score } \\
\text { All Subjects, } \\
\mathrm{n}=342\end{array}$ & $\begin{array}{c}\text { Excluding Subjects Taking } \\
\text { Antiinflammatory Drugs, } \\
\mathrm{n}=285\end{array}$ & $\begin{array}{c}\text { Excluding Subjects with } \\
\text { Chondrocalcinosis on } \\
\text { Radiography, } \mathrm{n}=312\end{array}$ \\
\hline < 4 Subregions* & 1.00 (reference) & 1.00 (reference) & 1.00 (reference) \\
$\geq$ 4 Subregions & $1.91(1.24,2.92)$ & $1.65(1.03,2.63)$ & $1.95(1.26,3.02)$ \\
< 6 Subregions** & 1.00 (reference) & 1.00 (reference) & 1.00 (reference) \\
$\geq$ 6 Subregions & $1.94(1.27,2.74)$ & $1.67(1.05,2.63)$ & $1.89(1.22,2.93)$ \\
\hline
\end{tabular}

\footnotetext{
* Knees with 4 or more subregions with cartilage score of 2 or above were considered to have widespread cartilage damage. ${ }^{* *}$ Knees with 6 or more subregions with cartilage score of 2 or above were considered to have widespread cartilage damage. $* * *$ Adjustment made for age, sex, and body mass index. ${ }^{\dagger}$ Synovitis score $\geq 1$ in at least 1 of 11 sites. CEMRI: contrast-enhanced magnetic resonance imaging.
}
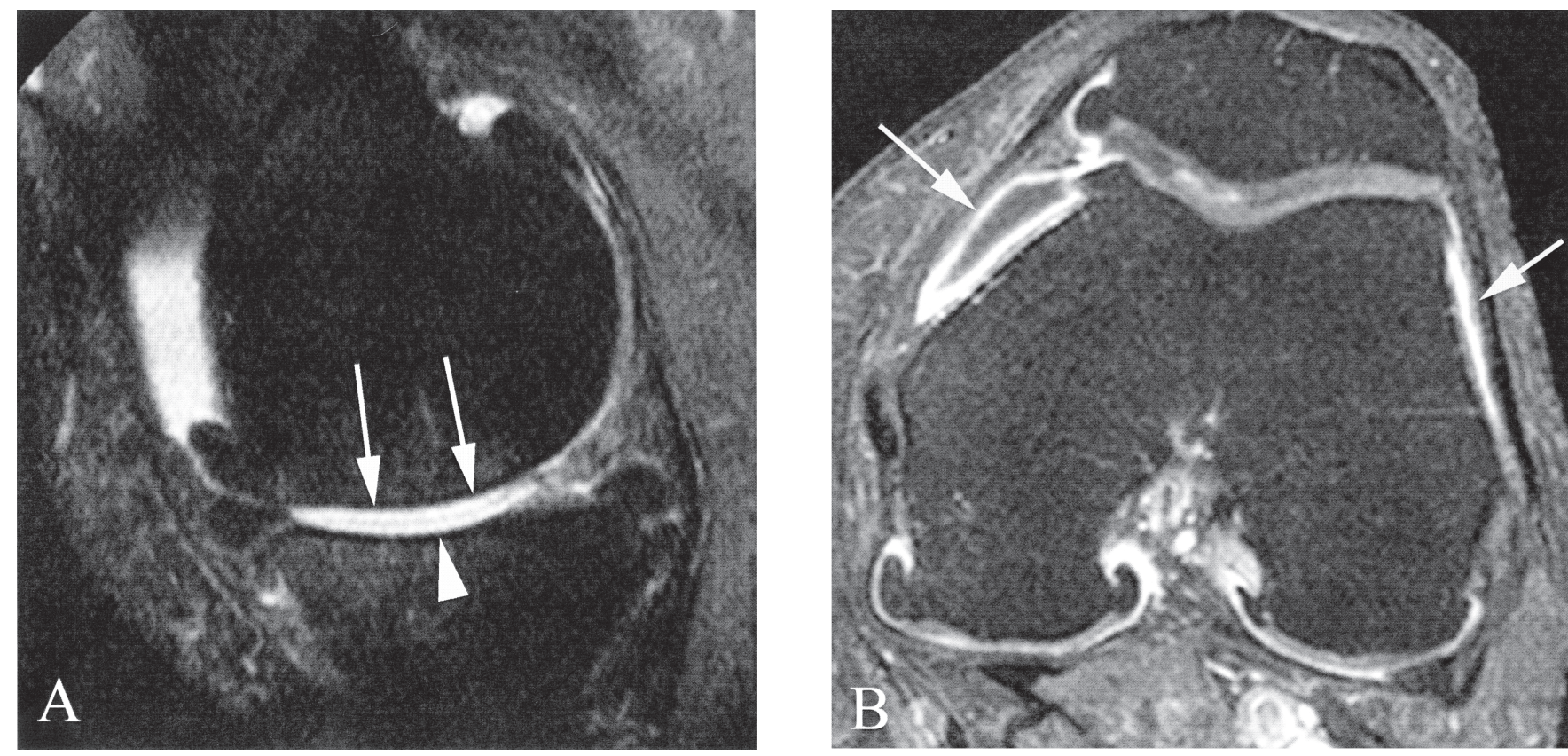

Figure 3. A. Sagittal unenhanced proton density-weighted 1.0T magnetic resonance imaging (MRI) shows diffuse full thickness cartilage loss at the central subregions of the medial femur (arrows) and tibia (arrowhead). Note anterior and posterior tibial and femoral osteophytes. In addition there is partial maceration of the posterior horn of the medial meniscus. B. Axial contrast-enhanced T1-weighted MRI of the same knee (acquired on the same day) shows moderate synovial thickening and enhancement at the medial and lateral patellar recesses (arrows). In addition there is joint effusion depicted as hypointensity (unenhanced) within the medial synovitis. 
Table 4. Cross-sectional association of meniscal damage and maximum synovitis scores from the whole knee.

\begin{tabular}{|c|c|c|c|}
\hline \multirow{2}{*}{$\begin{array}{l}\text { Max Meniscal } \\
\text { Grade in Any } \\
\text { Subregion }\end{array}$} & \multicolumn{3}{|c|}{ Adjusted* OR $\left(95 \%\right.$ CI) for CEMRI Max Synovitis Score ${ }^{\dagger}$} \\
\hline & $\begin{array}{l}\text { All Subjects, } \\
\quad n=343\end{array}$ & $\begin{array}{c}\text { Excluding Subjects Taking } \\
\text { Antiinflammatory Drugs, } \\
\qquad \mathrm{n}=286\end{array}$ & $\begin{array}{l}\text { Excluding Subjects with } \\
\text { Chondrocalcinosis on } \\
\text { Radiography, } \mathrm{n}=313\end{array}$ \\
\hline 0 & 1.00 (reference) & 1.00 (reference) & 1.00 (reference) \\
\hline$\geq 1$ & $0.88(0.52,1.49)$ & $1.47(0.94,2.29)$ & $1.24(0.81,1.9)$ \\
\hline 1 & $1.59(0.67,3.78)$ & $2.05(0.83,5.1)$ & $1.63(0.68,3.9)$ \\
\hline 2 & $0.87(0.50,1.52)$ & $1.1(0.6,2.03)$ & $0.86(0.47,1.57)$ \\
\hline$\geq 3$ & $1.74(0.99,3.04)$ & $1.65(0.9,3.03)$ & $1.49(0.84,2.66)$ \\
\hline
\end{tabular}

* Adjustment made for age, sex, and body mass index. ${ }^{\dagger}$ Synovitis score $\geq 1$ in at least 1 of 11 sites. CEMRI: contrast-enhanced magnetic resonance imaging.

assessed severity of synovitis (assessed based on the number of thickened villi visible on T2-weighted MRI) and severity of cartilage damage could not be demonstrated ${ }^{18}$. In that study, irrespective of cartilage damage severity, more than $80 \%$ of subjects did not have any synovitis. Although Meredith's analytic approach was very different from that of our study and thus a direct comparison of results is impossible, the reason that study did not find the association may be that their synovitis assessment was based on counting the number of visible villi, not a commonly used method to assess synovitis and one that does not necessarily represent the true extent of synovitis.

In our present study, we showed a borderline significant cross-sectional association between CEMRI-assessed whole-knee synovitis and severe meniscal damage. Mild or moderate meniscal damage was not associated with whole-knee synovitis. Grainger, et al used CEMRI to assess the relation between synovitis localized to the perimeniscal region and meniscal status ${ }^{19}$. They found that semiquantitatively assessed perimeniscal synovitis was not associated with meniscal tear. Although our study and that of Grainger, et al used different scoring systems, our results add to their findings and demonstrate a lack of statistically significant association between CEMRI-assessed synovitis and meniscal status, whether the synovitis was whole-knee or localized (i.e., perimeniscal).

Our study has several limitations that need to be mentioned. There is no histological validation of the CEMRI-based scoring system of synovitis. Blurring of CEMRI could have potentially affected the semiquantitative assessment. The selection process of the subjects for CEMRI study might have led to a cohort of subjects that does not represent typical patients with knee OA. The threshold values of synovial thickness for CEMRI-based grading of synovitis fail to take into account individual differences in the size and thickness of joint tissues in persons of different sizes. That could have confounded the analytic results. The non-CEMRI was acquired using $1.0 \mathrm{~T}$ system, while CEMRI was acquired using the $1.5 \mathrm{~T}$ system. This discrepancy in the strength of the magnetic field for scanners is a known limitation inherent to the MOST study design. However, a study has shown that semiquantitative assessment of knee OA features on both types of scanners are comparable ${ }^{20}$. Also, in the field of OA research, these systems are no longer state-of-the-art, and 3T systems are becoming increasingly more common. Use of a 3T system should be considered for future studies of synovitis in OA. Lastly, the cutoff values for the definition of "widespread cartilage damage" were arbitrary, and could be subject to debate. However, we used more than 1 definition and performed sensitivity analyses to alleviate this concern.

We used a comprehensive semiquantitative scoring system of synovitis in knee OA based on CEMRI, and demonstrated cross-sectional associations of whole-knee synovitis with radiographic OA and widespread cartilage damage, and borderline significant association with severe meniscal damage.

\section{ACKNOWLEDGMENT}

We thank the participants and staff of the MOST study at the clinical sites in Birmingham, Alabama, and Iowa City, Iowa, and at the Coordinating Center at the University of California San Francisco, San Francisco, California, USA.

\section{REFERENCES}

1. Myers SL, Flusser D, Brandt KD, Heck DA. Prevalence of cartilage shards in synovium and their association with synovitis in patients with early and end stage osteoarthritis. J Rheumatol 1992; 19:1247-51.

2. Scanzello CR, Umoh E, Pessler F, Diaz-Torne C, Miles T, Dicarlo $\mathrm{E}$, et al. Local cytokine profiles in knee osteoarthritis: elevated synovial fluid interleukin-15 differentiates early from end-stage disease. Osteoarthritis Cartilage 2009;17:1040-8.

3. Roemer FW, Zhang Y, Niu J, Lynch JA, Crema MD, Marra MD, et al. Tibiofemoral joint osteoarthritis: risk factors for MR-depicted fast cartilage loss over a 30-month period in the multicenter osteoarthritis study. Radiology 2009;252:772-80.

4. Ayral X, Pickering EH, Woodworth TG, Mackillop N, Dougados M. Synovitis: a potential predictive factor of structural progression of medial tibiofemoral knee osteoarthritis - results of a 1-year longitudinal arthroscopic study in 422 patients. Osteoarthritis Cartilage 2005;13:361-7.

5. Roeddecker K, Nagelschmidt M, Koebke J, Guensche K. Meniscal healing: a histological study in rabbits. Knee Surg Sports Traumatol

Personal non-commercial use only. The Journal of Rheumatology Copyright @ 2014 . All rights reserved. 
Arthrosc 1993;1:28-33.

6. Loeuille D, Sauliere N, Champigneulle J, Rat AC, Blum A, Chary-Valckenaere I. Comparing non-enhanced and enhanced sequences in the assessment of effusion and synovitis in knee OA: associations with clinical, macroscopic and microscopic features. Osteoarthritis Cartilage 2011;19:1433-9.

7. Guermazi A, Roemer FW, Hayashi D, Crema MD, Niu J, Zhang Y, et al. Assessment of synovitis with contrast-enhanced MRI using a whole-joint semiquantitative scoring system in people with, or at high risk of, knee osteoarthritis: the MOST study. Ann Rheum Dis 2011;70:805-11.

8. Roemer FW, Guermazi A, Hunter DJ, Niu J, Zhang Y, Englund M, et al. The association of meniscal damage with joint effusion in persons without radiographic osteoarthritis: the Framingham and MOST osteoarthritis studies. Osteoarthritis Cartilage 2009; 17:748-53.

9. Krasnokutsky S, Belitskaya-Levy I, Bencardino J, Samuels J, Attur $\mathrm{M}$, Regatte R, et al. Quantitative magnetic resonance imaging evidence of synovial proliferation is associated with radiographic severity of knee osteoarthritis. Arthritis Rheum 2011;63:2983-91.

10. Felson DT, Niu J, Guermazi A, Roemer F, Aliabadi P, Clancy M, et al. Correlation of the development of knee pain with enlarging bone marrow lesions on magnetic resonance imaging. Arthritis Rheum 2007;56:2986-92.

11. Peterfy CG, Guermazi A, Zaim PF. Non-fluoroscopic method for flexed radiography of the knee that allows reproducible joint-space width measurement. Arthritis Rheum 1998;41:S361.

12. Kellgren JH, Lawrence JS. Radiological assessment of osteo-arthrosis. Ann Rheum Dis 1957;16:494-502.

13. Ostergaard M, Klarlund M. Importance of timing of post-contrast MRI in rheumatoid arthritis: what happens during the first 60 minutes after IV gadolinium-DTPA? Ann Rheum Dis 2001;60:1050-4.
14. Peterfy CG, Guermazi A, Zaim S, Tirman PF, Miaux Y, White D, et al. Whole-Organ Magnetic Resonance Imaging Score (WORMS) of the knee in osteoarthritis. Osteoarthritis Cartilage 2004;12:177-90.

15. Aigner T, Sachse A, Gebhard PM, Roach HI. Osteoarthritis: pathobiology-targets and ways for therapeutic intervention. Adv Drug Deliv Rev 2006;58:128-49.

16. Scanzello CR, McKeon B, Swaim BH, DiCarlo E, Asomugha EU, Kanda V, et al. Synovial inflammation in patients undergoing arthroscopic meniscectomy: molecular characterization and relationship to symptoms. Arthritis Rheum 2011;63:391-400.

17. Baker KR, Matthan NR, Lichtenstein AH, Niu J, Guermazi A, Roemer F, et al. Association of plasma n- 6 and n-3 polyunsaturated fatty acids with synovitis in the knee: the MOST study. Osteoarthritis Cartilage 2012;20:382-7.

18. Meredith DS, Losina E, Neumann G, Yoshioka H, Lang PK, Katz JN. Empirical evaluation of the inter-relationship of articular elements involved in the pathoanatomy of knee osteoarthritis using magnetic resonance imaging. BMC Musculoskelet Disord 2009; 10:133.

19. Grainger AJ, Rhodes LA, Keenan AM, Emery P, Conaghan PG. Quantifying peri-meniscal synovitis and its relationship to meniscal pathology in osteoarthritis of the knee. Eur Radiol 2007;17:119-24.

20. Roemer FW, Lynch JA, Niu J, Zhang Y, Crema MD, Tolstykh I, et al. A comparison of dedicated 1.0 T extremity MRI vs large-bore 1.5 T MRI for semiquantitative whole organ assessment of osteoarthritis: the MOST study. Osteoarthritis Cartilage 2010;18:168-74. 